\title{
Novel Intensity-demodulated Fiber-optic Refractive Index Sensor Based on Splicing Point Tapered Fiber
}

\author{
Liangquan Zhu, ${ }^{\text {\# }}$, Qijing Lin, ${ }^{1,2,3,4 \#}$ Fuzheng Zhang, ${ }^{2}$ Feng Han, ${ }^{1,2 *}$ \\ Man Zhao, ${ }^{2}$ Ping Yang, ${ }^{2}$ and Zhuangde Jiang ${ }^{2}$ \\ ${ }^{1}$ State Key Laboratory of Mechanical Manufacturing Systems Engineering, Xi'an Jiaotong University, \\ Beilin district, Xi'an 710049, China \\ ${ }^{2}$ Collaborative Innovation Center of High-End Manufacturing Equipment, Xi'an Jiaotong University, \\ Qujiang new district, Xi'an 710054, China \\ ${ }^{3}$ Xi'an Jiaotong University Suzhou Institute, Industrial park, Suzhou 215123, China \\ ${ }^{4}$ State Key Laboratory of Mechanical System and Vibration, Shanghai Jiaotong University, \\ Minhang district, Shanghai 200240, China
}

(Received March 29, 2021; accepted May 17, 2021)

Keywords: refractive index sensor, fiber-optic sensors, Michelson interferometer, splicing point tapered fiber

A novel fiber-optic refractive index (RI) sensor based on a splicing point tapered fiber Michelson interferometer is proposed. It consists of a short section of thin-core fiber (TCF), which is spliced to a standard single-mode fiber, then the fused region is drawn into a taper by using residual heat. The interference between the core and cladding modes in the TCF is utilized, which is insensitive to variations in the RI of the surrounding environment but susceptible to external temperature changes. However, owing to the Fresnel reflection on the fiber end face, the intensity of the reflection spectrum decreases with increasing RI of the surrounding environment. Thus, the RI and temperature can be demodulated by the intensity and wavelength of the reflection spectrum, respectively, which can eliminate the crosstalk between RI and temperature. We experimentally found that the sensor has RI sensitivity of up to $68.60 \mathrm{~dB} / \mathrm{RIU}$ for a TCF length of $10 \mathrm{~mm}$ and a waist diameter of $50 \mu \mathrm{m}$. The temperature characteristics of the sensor in the range of 30 to $90{ }^{\circ} \mathrm{C}$ were also investigated, and a sensitivity of about $57 \mathrm{pm} /{ }^{\circ} \mathrm{C}$ was obtained. Moreover, the compact size, easy fabrication, and low cost of the proposed fiber-optic RI sensor make it an attractive device.

\section{Introduction}

Owing to the advantages of inexpensiveness, compactness, high precision, high sensitivity, and immunity to electromagnetic waves, ${ }^{(1,2)}$ fiber-optic sensors potentially have a broad range of applications in the sensing field, especially for sensing applications in harsh (conductive, explosive, or corrosive) environments. Therefore, fiber-optic sensors have been widely used to measure quantities such as temperature, ${ }^{(2,3)}$ strain, ${ }^{(3,4)}$ vibration, ${ }^{(5,6)}$ humidity, ${ }^{(7,8)}$ and pressure. ${ }^{(9,10)}$ In particular, many RI fiber-optic sensors have been reported. ${ }^{(11-14)}$

*Corresponding author: e-mail: xjjingmi@163.com

\#These authors contributed equally to this work.

https://doi.org/10.18494/SAM.2021.3254 
Measurement of the RI of the surrounding environment has a wide range of applications in chemical analysis, biomedical research, environmental monitoring and surveillance, food manufacturing, and other fields. Therefore, various fiber structures have been used to fabricate RI sensors such as a Mach-Zehnder interferometer, ${ }^{(12,15)}$ Sagnac interferometer, ${ }^{(14,16)}$ FabryPerot interferometer (FPI), ${ }^{(11)}$ and Michelson interferometer (MI). ${ }^{(17-21)}$ In recent years, RI sensors based on an MI have been widely reported due to their compact structure, easy fabrication, and low cost. Furthermore, these RI sensors are fabricated using different fabrication processes, such as coating, ${ }^{(19)}$ side polishing, ${ }^{(22)}$ and $\mathrm{CO}_{2}$ laser irradiation, ${ }^{(23)}$ while some sensors use specialty fibers such as a dual-core fiber $(\mathrm{DCF}){ }^{(18)}$ a double-clad fiber, ${ }^{(24)}$ and a thin-core fiber (TCF). ${ }^{(21)}$ In addition, different structures (U shape, ${ }^{(14)}$ tapered shape, ${ }^{(20,25)}$ etc.) and special fusion methods (core offset, ${ }^{(17)}$ waist-enlarged fiber taper, ${ }^{(26)}$ etc.) are also adopted to acquire high sensitivity. Although some structures can provide acceptable sensitivity, problems still exist such as fragility, complex preprocessing, and the requirement of expensive femtosecond lasers or high-precision taper facilities, which all significantly limit the practical applicability of the sensors. In addition, variation of the temperature will also cause a wavelength shift of the fiberoptic sensors. When wavelength demodulation is used in RI measurement, the temperature changes can introduce the problem of crosstalk. Therefore, it is necessary to fabricate a fiberoptic RI sensor that can eliminate crosstalk between temperature and RI.

Because different demodulation methods can be used to measure different parameters to effectively solve the problem of crosstalk between multiple parameters, many researchers have begun to use intensity and wavelength demodulation to separately measure dual physical parameters. One of the multi-parameter sensing technologies is based on a combination of an MI and the effect of Fresnel reflection, which can be effectively used to separately measure temperature and RI, and eliminate crosstalk between them. ${ }^{(24,27,28)}$ Zhao et al. ${ }^{(28)}$ fabricated an MI by cascading a single-mode fiber (SMF), a multi-mode fiber (MMF), and another SMF for the detection of temperature and RI. The highest sensitivities of the temperature and RI were reported to be $92.6 \mathrm{pm} /{ }^{\circ} \mathrm{C}$ and $67.9 \mathrm{~dB} / \mathrm{RIU}$, respectively, in the RI range of 1.33 to 1.3737 . In addition, Musa et al. ${ }^{(24)}$ fabricated a fiber sensor with an SMF-MMF-DCF structure for the simultaneous measurement of temperature and RI. The sensitivity of the RI sensor was calculated to be about 22 and $5 \mathrm{~dB} / \mathrm{RIU}$ at the RIs of 1.30 and 1.38, respectively. Although high sensitivity and the elimination of the effect of temperature crosstalk were achieved in these studies, the complicated structure of the sensors would make them difficult to use in practical application. Therefore, a fiber-optic RI sensor with a simple manufacturing process and high sensitivity is needed.

In this study, a novel fiber structure based on a splicing point tapered fiber-optic MI is proposed and experimentally verified. The sensing head is fabricated by using a short section of a TCF spliced to an SMF, with the splicing point drawn into a taper by a splice machine. The tapered structure at the splicing point can induce more light to enter the cladding of the TCF to excite higher order cladding modes, ${ }^{(25,29)}$ thus achieving the coupling of the fiber core mode and cladding modes. In addition, the fiber structure only requires one fusion splicing, which can reduce the likelihood of operation errors caused by multiple fusion splicing and does not require expensive and precise instruments. Moreover, the fiber structure can realize the demodulation of 
temperature and refractive index by the wavelength and intensity, respectively, so as to eliminate the interference caused by the temperature in the process of RI measurement. The proposed RI fiber-optic sensor also has the advantages of a simple fabrication process and low cost.

\section{Design and Principle}

As depicted in Fig. 1(a), light from a broadband light source (BBS, ASE-CL, Max-ray, Hefei, China) passing through a circulator then through a sensor is reflected back at the cleaved ends of the fiber. After that, the reflected light, after passing through the circulator again, is finally monitored by an optical spectrum analyzer (OSA, MS9740A, Anritsu, Japan) and recorded as a reference. When light in the SMF is transmitted into the tapered splicing point, because the fiber diameter decreases, part of the light will couple into the fiber cladding, which will be transmitted at the same time as the light in the fiber core, as shown in Fig. 1(b). In addition, when they reach the cleaved ends of the TCF, due to Fresnel reflection, they will be reflected and recoupled into the fiber core at the tapered splicing point. Consequently, a sensor based on Michelson interference is realized, and visible interference fringes can be observed in the OSA.

A schematic diagram and a microscopy image of the tapered splicing point, which was fabricated by splicing a short section of a TCF (Corning HI 1060Flex) with a standard SMF, then drawing the fused region into a taper using residual heat, are shown in Figs. 1(b) and 1(c), respectively. The core and cladding diameters of the TCF are 4 and $125 \mu \mathrm{m}$, respectively. Owing to the core mismatch and the tapered region at the splicing point, more of the light can enter the cladding to form cladding modes and obtain a clearer interference fringe. The proposed structure was fabricated using a commercial splicer (Fitel, S178), which provides an accurate discharge intensity and control of the motor.

The BeamPROP method ${ }^{(30)}$ was used to simulate the electric field distribution of the proposed structure. As shown in Fig. 2(a), owing to the core mismatch of the TCF and SMF, the light in the

(a)

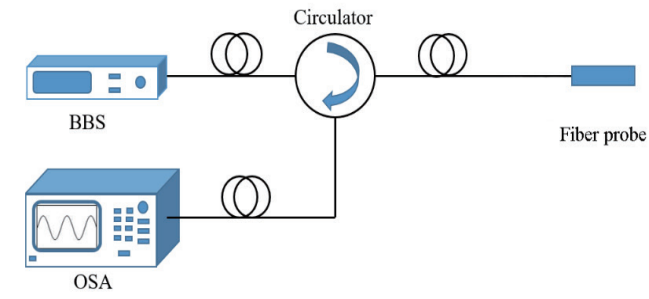

(b)

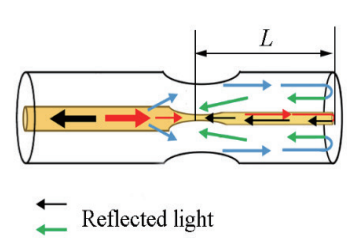

(c)

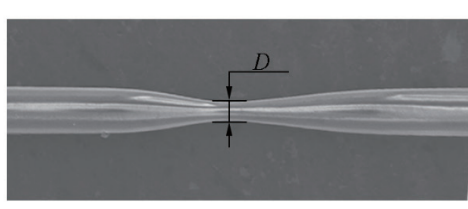

Fig. 1. (Color online) (a) Schematic structure of investigated fiber-optic interferometer sensing setup; (b) schematic of fiber-optic sensor; and (c) microscopy image of the tapered splicing point. 

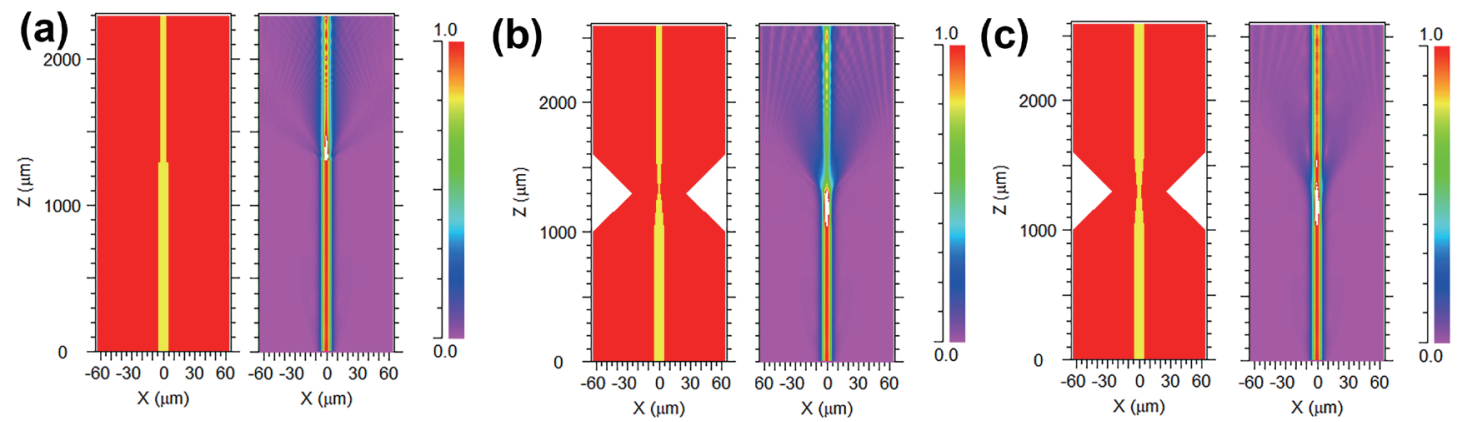

Fig. 2. (Color online) Simulation model diagram (XZ view) and simulated electric field distribution of (a) SMFTCF structure, (b) SMF-TCF structure with a taper $(50 \mu \mathrm{m})$ in the splicing point; and (c) SMF structure with a taper $(50 \mu \mathrm{m})$.

fiber core of the SMF leaks into the cladding of the TCF, thereby forming an electric field distribution in the cladding. However, the electric field distribution in the cladding of the TCF is weak. To increase the amount of light coupled into the fiber cladding, the splicing point was drawn into a taper as depicted in Fig. 2(b). It can be seen that with decreasing diameter of the splicing point, more light leaks into the cladding of the TCF to form a stronger electric field distribution. In addition, compared with the same taper $(50 \mu \mathrm{m})$ in the SMF [Fig. 2(c)], the electric field distribution in the cladding of the TCF is clearer, resulting in clearer interference fringes in the proposed structure. In addition, since Fresnel reflection occurs on the end face of the fiber, more light is lost. Thus, to fabricate a fiber-optic MI sensor, it is necessary to induce more light to couple into the cladding.

According to the mode interference theory, when interference occurs between the core and cladding modes, the interference pattern intensity can be expressed as ${ }^{(20,24,25)}$

$$
I=\left(I_{\text {core }}+\sum_{m} I_{\text {cladding }}^{m}+\sum_{m} 2 \sqrt{I_{\text {core }} I_{\text {cladding }}^{m}} \cos \left(\Delta \varphi^{m}\right)\right) \cdot R,
$$

where $I_{\text {core }}$ and $I_{\text {cladding }}^{m}$ are the intensities of the fundamental and $m$ th-order cladding modes, respectively, and $R$ is the reflectivity at the end of the fiber. Owing to the different effective RIs of the core and cladding in the TCF, the accumulated optical phase of the core and cladding modes can be calculated as

$$
\Delta \varphi^{m}=4 \pi\left(n_{\text {core }}-n_{\text {cladding }}^{m}\right) \frac{L}{\lambda_{m}}=\frac{4 \pi \Delta n_{\text {eff }}^{m} L}{\lambda_{m}},
$$

where $n_{\text {core }}$ and $n_{\text {cladding }}^{m}$ are the RIs of the fiber core and cladding respectively, $\Delta n_{\text {eff }}^{m}$ is the difference between the RI of the fiber core and that of the $m$ th-order cladding mode, $\lambda_{m}$ is the wavelength of the attenuation peaks in vacuum, and $L$ is the interference length. From Eqs. (1) and (2), it can be seen that the maximum attenuation will appear at 


$$
\lambda_{m}=\frac{4 \pi \Delta n_{e f f}^{m} L}{(2 k+1) \pi}
$$

where $k$ is an integer. When the RIs of the surrounding environment changes, the effective RI difference $\Delta n_{e f f}^{m}$ will change to $\delta n_{\text {eff }}^{m}$, leading to the following change in wavelength:

$$
\delta \lambda_{m}=\frac{4 \delta n_{e f f}^{m} L}{2 k+1} .
$$

Furthermore, according to the theory of Fresnel reflection, at the end face of the fiber probe, the reflectivity can be given by $(28)$

$$
R=\frac{\left(n_{s i}-n_{s o}\right)^{2}}{\left(n_{s i}+n_{s o}\right)^{2}}
$$

where $n_{s i}$ and $n_{s o}$ are the RIs of the fiber and the surrounding environment, respectively. According to Eqs. (1) and (5), the intensity of the interference pattern is strongly dependent on the RI of the surrounding environment. Therefore, the proposed splicing point with the tapered structure can act as an intensity demodulation sensor for RI measurement. From the previous analysis, as the RI of the surrounding environment decreases, the intensity of the reflection spectrum gradually increases and shifts to a longer wavelength.

Owing to the thermal expansion effect and the thermo-optic effect, the temperature variation can induce changes in the length and the effective RIs of the TCF, resulting in a shift in the interference fringes. From Eqs. (2) and (3), the temperature sensitivity can be expressed by (31)

$$
\frac{d \lambda_{m}}{d T}=\left[\frac{\lambda_{m}}{\Delta n_{\text {eff }}^{m}}\left(\frac{d n_{\text {core }}}{d T}-\frac{d n_{\text {caldding }}^{m}}{d T}\right)+\frac{\lambda_{m}}{L} \cdot \frac{d L}{d T}\right] /\left(1-\frac{\lambda_{m}}{\Delta n_{\text {eff }}^{m}} \frac{\partial \Delta n_{\text {eff }}^{m}}{\partial T}\right) .
$$

It can be seen from Eq. (6) that the sensitivity depends on the variations of the interference length and the effective RIs of the fiber core and cladding with the temperature.

\section{Experiment and Discussion}

\subsection{Fabrication of RI sensor}

From the previous discussion, the excited cladding mode will interfere with the fiber core mode, and interference fringes will appear in the reflection spectrum. Thus, a fiber probe with a TCF length of $10 \mathrm{~mm}$ and a splicing point with a waist diameter of $50 \mu \mathrm{m}$ was fabricated. A comparison of the reflection spectrum of the fiber probe and the light source transmission 
spectrum is shown in Fig. 3(a). The light source transmission spectrum was obtained by directly connecting the light source (BBS, ASE-CL, 100-1000 mW, Hefei, China) to the spectrometer when the output power of the light source was $300 \mathrm{~mW}$. Compared with the light source spectrum, there are clearer interference fringes in the reflection spectrum of the optical fiber probe. However, owing to the noise in the light source, the light source spectrum is not flat. To distinguish the light source noise and interference fringes, a fast Fourier transform (FFT) of the spectra in Fig. 3(a) was carried out to obtain the spatial frequency spectra [Fig. 3(b)]. It can be inferred from the spatial frequency spectra that the first three peaks were induced by the noise in the light source and the other three peaks were caused by fiber mode interference. Thus, to eliminate the noise from the light source, the spectra were filtered by an FFT filter, and the filtered spectra are shown in Fig. 3(c). It can be seen that after FFT filtering, the transmission spectrum of the light source is flat and the interference fringes of the reflection spectrum of the fiber probe are clearly visible.

\subsection{Response to RI}

To determine the sensitivity of the RI response measurement, we prepared seven salt solutions with different volume concentrations and corresponding RIs of 1.333, 1.3399, 1.3460, $1.3529,1.36,1.3672$, and 1.3781. Then, the MI was mounted on a fiber holder and immersed in each salt solution to the same depth. The experimental setup is shown in Figs. 4(a) and 4(b). After each measurement, to eliminate the influence of residue adhering on the fiber surface, the fiber probe was soaked in pure water for $3 \mathrm{~min}$, then washed with pure water five times. The measured wavelength shift of the reflection spectra of the sensor is illustrated in Fig. 4(c). The reflection spectra, after FFT filtering, are shown in Fig. 4(d). Among them, the interference fringes in the wavelength range of 1594-1608 nm are shown in Fig. 4(e). It can be observed that as the RI increases, the intensity of the reflection spectra rapidly decreases while the spectra hardly shift to shorter wavelengths. Moreover, as shown in Fig. 4(f), the intensity variation of the reflection spectra with RIs in the range of 1.333-1.3781 shows good agreement with the linear fitting curves, with a coefficient of determination $\left(R^{2}\right)$ of 0.993 . As described earlier, the changes in RI result in wavelength shifts and intensity variation of the transmission spectrum. It is clear

(a)

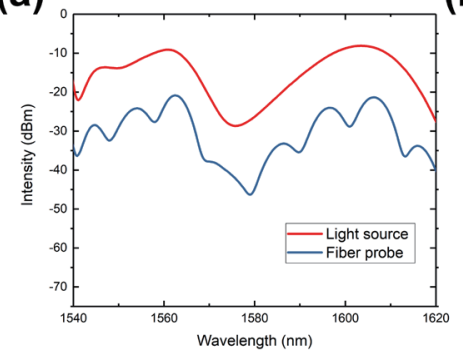

(b)

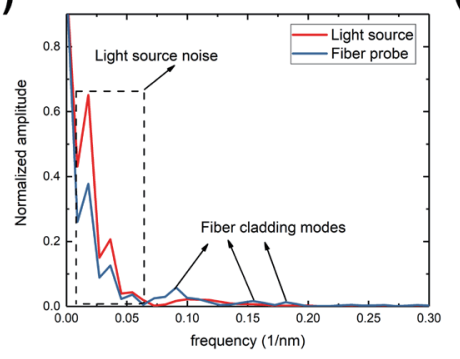

(c)

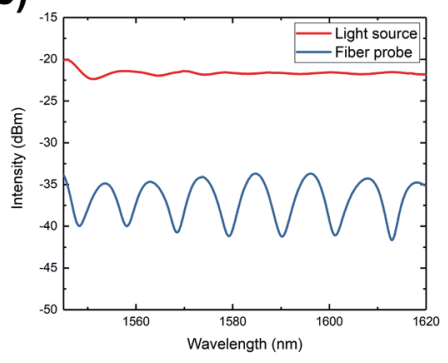

Fig. 3. (Color online) (a) Spectra of the light source and the fiber probe with the TCF length of $10 \mathrm{~mm}$ and the waist diameter of $50 \mu \mathrm{m}$; (b) spatial frequency spectra of light source and fiber probe; and (c) spectra of the light source and the fiber probe after FFT filtering. 
(a)

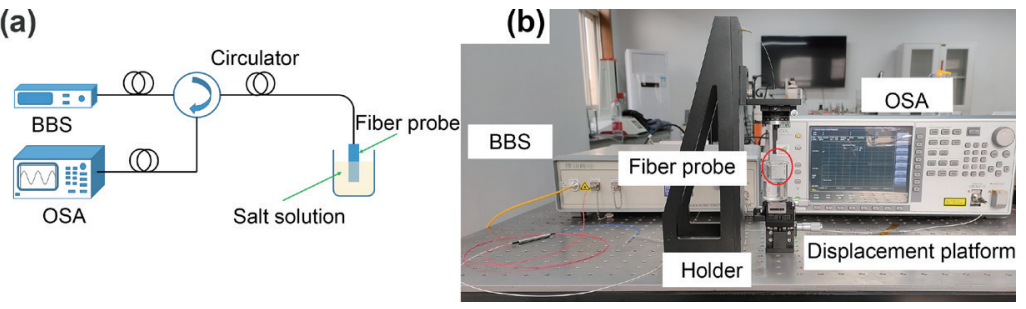

(c)

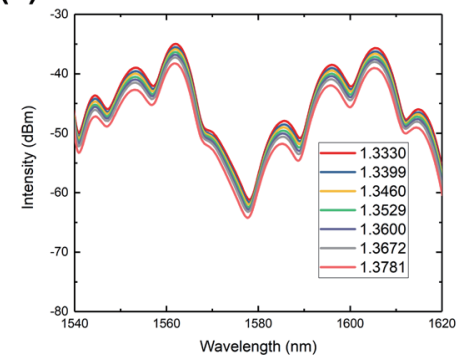

(d)

(e)
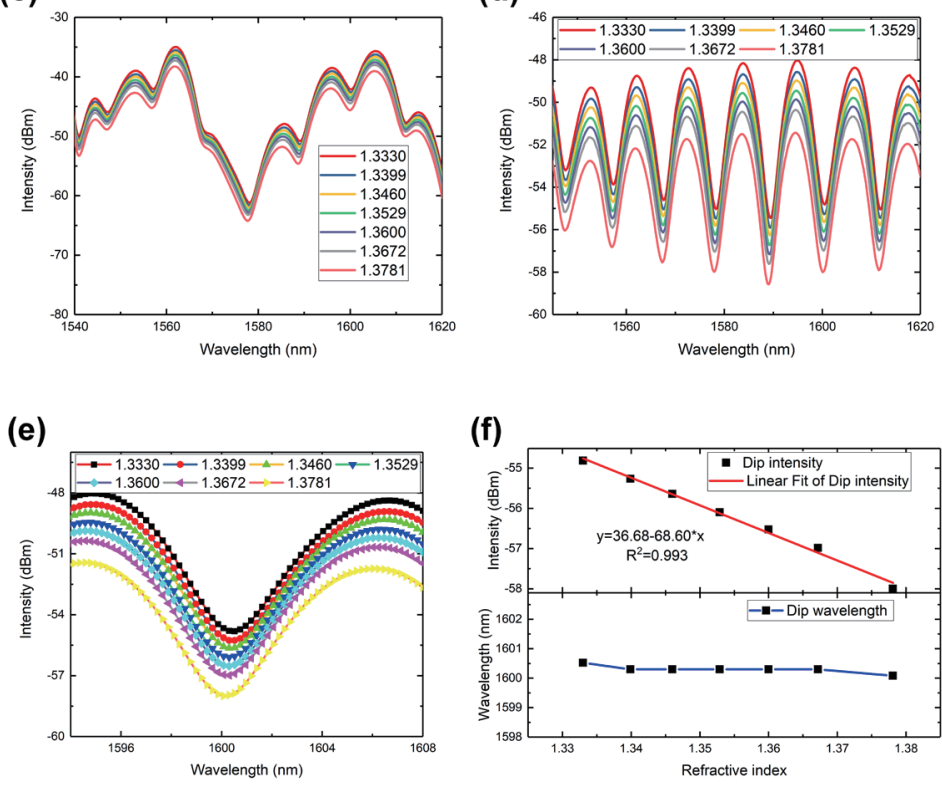

(f)

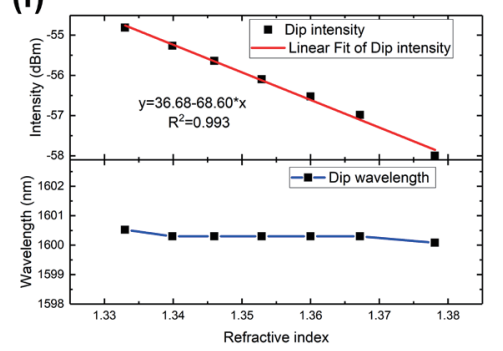

Fig. 4. (Color online) (a) Schematic of experimental setfup for the RI measurement; (b) experimental setup for the RI measurement; (c) measured reflection spectra at different RIs; (d) reflection spectra at different RIs after FFT filtering; (e) interference fringes of reflection spectra in the wavelength range of 1594-1608 nm; and (e) and (f) wavelength shifts and intensity changes of the transmission dip versus RI.

that the proposed sensor is more suitable for use as an intensity-modulated sensor than as a wavelength-modulated sensor. The sensitivity of the RI sensor was calculated to be about 68.60 $\mathrm{dB} / \mathrm{RIU}$ in the RI range of 1.333 to 1.3781 .

\subsection{Response to temperature}

As the RI in the surrounding environment changes with the temperature, it is difficult to distinguish whether the change in RI is caused by changes in temperature or by changes in the surrounding environment. Therefore, temperature variation affects the RI measurement and its sensitivity. To obtain the response characteristics to temperature, the sensor was inserted in a furnace and fixed by high-temperature air-set cement (OB-600), as depicted in Figs. 5(a) and 5(b). By controlling the furnace, the temperature was increased from 30 to $90{ }^{\circ} \mathrm{C}$ in steps of 10 ${ }^{\circ} \mathrm{C}$. At each temperature, to acquire the reflection spectra accurately, the retention time was set to $5 \mathrm{~min}$, which was long enough to obtain a stable temperature. Figure 5(c) shows the 
(a)

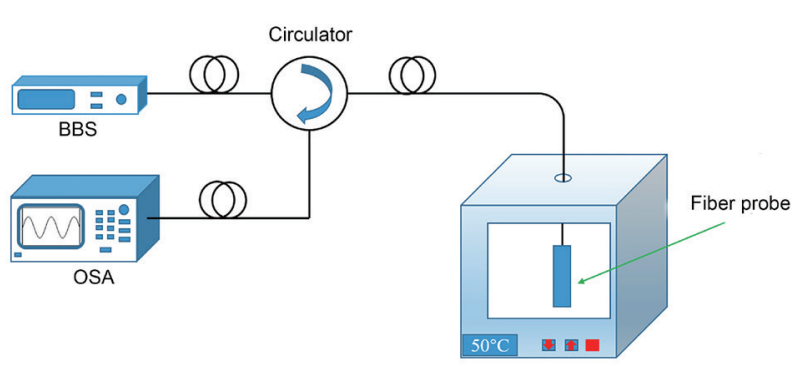

(b)

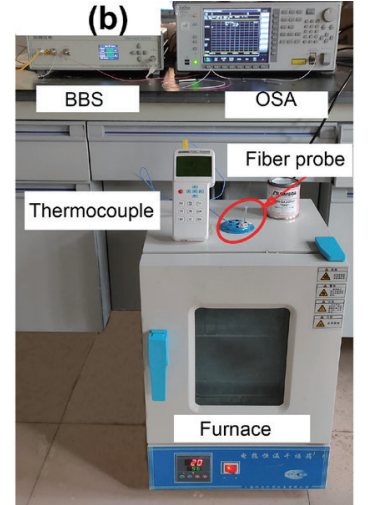

(c)

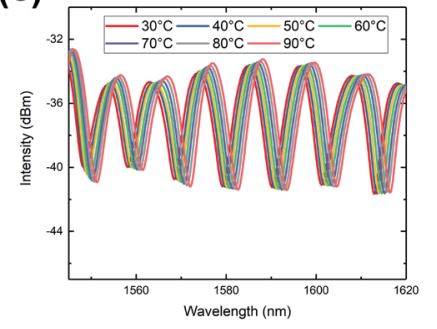

(d)

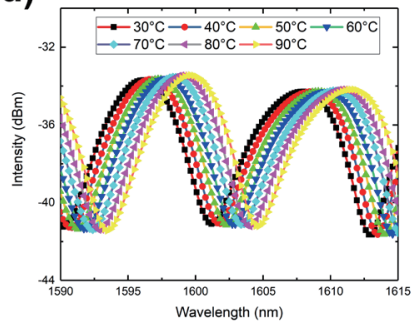

(e)

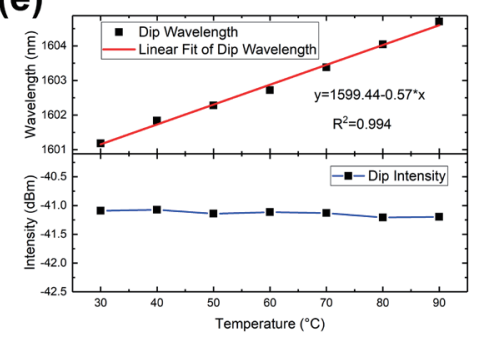

Fig. 5. (Color online) (a) Schematic of experimental setup for the temperature measurement; (b) experimental setup for the temperature measurement; (c) reflection spectra after FFT filtering at different temperatures; (d) corresponding interference fringes in the wavelength range of 1590-1615nm; and (e) wavelength shifts and intensity changes of the interference fringes versus temperature.

corresponding reflection spectra after FFT filtering at different temperatures, and Fig. 5(d) shows the interference fringes of the reflection spectra in the wavelength range of 1590-1615 $\mathrm{nm}$. As the temperature increases, the interference fringes shift to a longer wavelength. It can be inferred from Fig. 5(e) that the reflection spectra fringes shift linearly with a sensitivity of about $57 \mathrm{pm} /{ }^{\circ} \mathrm{C}$. In addition, the intensity of interference fringes hardly changes with the temperature.

\subsection{Comparison with previous RI sensors}

As the RI and temperature are separately demodulated by intensity and wavelength, respectively, the simultaneous measurement of temperature and RI can be easily achieved, so as to solve the crosstalk problem caused by temperature changes in the RI measurement. A comparison of optical fiber RI sensors is shown in Table 1. It can be seen that the FPI structures have the highest RI sensitivity. However, the length of the FP cavity is usually on the micrometer level, so the fabrication requires micromanipulation and the fabrication process is complicated, limiting the application of these sensors. Compared with these structures, our sensor structure is easy to fabricate and only requires a fusion splicer. In addition, in the RI range of 1.333 to 1.3781 , the intensity variation of our structure shows good agreement with the linear fitting. Thus, compared with other structures, the fiber structure is easier to demodulate. Furthermore, the proposed structure has relatively high temperature sensitivity compared with the other structures. As the RI of a liquid varies with the temperature, the higher temperature sensitivity can better compensate for the influence of temperature on the RI. 
Table 1

Comparison with other fiber-optic RI sensors.

\begin{tabular}{lcccccc}
\hline Structure & Fabrication method & Range & $\begin{array}{c}\text { RI sensitivity } \\
(\mathrm{dB} / \mathrm{RIU})\end{array}$ & $\begin{array}{c}\text { Temperature } \\
\text { sensitivity }\left(\mathrm{pm} /{ }^{\circ} \mathrm{C}\right)\end{array}$ & Linearity & Ref. \\
\hline LPFG & $\begin{array}{c}\text { Inscribe fiber grating and } \\
\text { deposit carbon nanotube }\end{array}$ & $1.33-1.38$ & 31 & Linear & 32 \\
\hline FPI & $\begin{array}{c}\text { Femtosecond laser } \\
\text { micromachining }\end{array}$ & $1.36-1.42$ & 110 & Nonlinear & 11 \\
\hline FPI & $\begin{array}{c}\text { Splice SMF-capillary tube } \\
(\mu \mathrm{m})-\text { SMF }\end{array}$ & $1.30-1.432$ & $216.37(1.30)$ & 10.85 & Nonlinear & 33 \\
\hline FPI & Splice $50 \mu \mathrm{m}$ TCF with SMF & $1.3326-1.4305$ & $1111(1.4305)$ & 10.1 & Nonlinear & 34 \\
\hline MI & Splice SMF-MMF-SMF & $1.33-1.3737$ & $67.98(1.3737)$ & 92.6 & Nonlinear & 28 \\
\hline MI & Splice SMF-MMF-DCF & $1.30-1.38$ & 22 & 3.7 & Nonlinear & 24 \\
\hline MI & $\begin{array}{c}\text { Stretch SMF to 30 } \mu \mathrm{m} \\
\text { microfiber }\end{array}$ & $1.3329-1.3904$ & 112.2 & 12 & Nonlinear & 35 \\
\hline MI & $\begin{array}{c}\text { Splice microfiber to SMF } \\
\text { on Multicore fiber }\end{array}$ & $1.31-1.39$ & 74.2 & 9.75 & Nonlinear & 36 \\
\hline $\begin{array}{l}\text { This paper } \\
(\mathrm{MI})\end{array}$ & $\begin{array}{c}\text { Splice SMF to TCF } \\
\text { and taper using splicer }\end{array}$ & $1.333-1.3781$ & 68.60 & 57 & Linear & - \\
\hline
\end{tabular}

\section{Conclusion}

A novel compact RI fiber-optic sensor based on an MI was proposed and experimentally demonstrated. The sensor was formed by splicing a short section of a TCF into an SMF, then the fused region was drawn into the taper by using residual heat. The intensity-modulation RI sensor exhibited a sensitivity of up to $68.60 \mathrm{~dB} / \mathrm{RIU}$, and it was observed that the wavelength of the transmission spectrum hardly changed with the RI. The temperature characteristic of the sensor was also investigated, and a sensitivity of about $57 \mathrm{pm} /{ }^{\circ} \mathrm{C}$ was observed. Owing to the use of intensity and wavelength to demodulate the RI and temperature, respectively, the fiber interferometer can act as a high-accuracy RI sensor.

\section{Acknowledgments}

The authors are grateful for the financial support from National Key R\&D Program of China (No.2018YFF0212301), National Natural Science Foundation of China (Nos. 51805421 and 91748207), Natural Science Foundation of Jiangsu Province (No. BK20180238). We also appreciate the support from the International Joint Laboratory for Micro/Nano Manufacturing and Measurement Technologies.

\section{References}

1 F. Zhang, Q. Lin, L. Zhu, N. Zhao, F. Han, L. Zhao, and Z. Jiang: Materials 13 (2020) 5475. https://doi. org/10.3390/ma13235475

2 R. Subramanian, C. Zhu, H. Zhao, and H. Li: IEEE Photonics Technol. Lett. 30 (2018) 327. https://doi. org/10.1109/LPT.2017.2787157

3 F. Zhang, N. Zhao, Q. Lin, Z. Wu, B. Tian, P. Shi, P. Yang, and Z. Jiang: AIP Adv. 10 (2020). https://doi. org/10.1063/5.0005151

4 H. A. Rahman, H. R. A. Rahim, S. W. Harun, M. Yasin, R. Apsari, H. Ahmad, and W. A. B. Wan Abas: Opt. Laser Technol. 45 (2013) 336. https://doi.org/10.1016/j.optlastec.2012.06.027 
5 J. Villatoro, E. Antonio-Lopez, A. Schülzgen, and R. Amezcua-Correa: Opt. Lett. 42 (2017) 2022. https://doi. org/10.1364/ol.42.002022

6 A. Masoudi, J. A. Pilgrim, T. P. Newson, and G. Brambilla: J. Light. Technol. 37 (2019) 1352. https://doi. org/10.1109/JLT.2019.2893038

7 D. Lopez-Torres, C. Elosua, J. Villatoro, J. Zubia, M. Rothhardt, K. Schuster, and F. J. Arregui: Sens. Actuators, B 251 (2017)1059. https://doi.org/10.1016/j.snb.2017.05.125

8 Y. Zhao, R. J. Tong, M. Q. Chen, and F. Xia: Sens. Actuators, B 284 (2019) 96. https://doi.org/10.1016/j. $\underline{\text { snb.2018.12.130 }}$

9 X. Qi, S. Wang, J. Jiang, K. Liu, X. Wang, Y. Yang, and T. Liu: J. Light. Technol. 37 (2019) 2719. https://doi. org/10.1109/JLT.2018.2876717

10 Q. Zhang, J. Lei, Y. Chen, Y. Wu, C. Chen, and H. Xiao: IEEE Sens. J. 19 (2019) 11242. https://doi.org/10.1109/ JSEN.2019.2935689

11 Z. L. Ran, Y. J. Rao, W. J. Liu, X. Liao, and K. S. Chiang: Opt. Express 16 (2008) 2252. https://doi.org/10.1364/ OE.16.002252

12 F. Zhang, N. Zhao, Q. Lin, L. Zhu, F. Han, L. Zhao, P. Yang, and Z. Jiang: United States: Proc. 2020 IEEE 15th Int. Conf. Nano/Micro Engineered and Molecular System (IEEE, 2020) 27-30.

13 J. Liu, M. Wang, X. Liang, Y. Dong, H. Xiao, and S. Jian: Opt. Laser Technol. 93 (2017) 74. https://doi. org/10.1016/j.optlastec.2017.02.008

14 Y. Zhao, X. Liu, R. Q. Lv, and Q. Wang: Sens. Actuators, B 243 (2017) 800. https://doi.org/10.1016/j. $\underline{\text { snb.2016.12.016 }}$

15 Q. Yao, H. Meng, W. Wang, H. Xue, R. Xiong, B. Huang, C. Tan, and X. Huang: Sens. Actuators, A 209 (2014) 73. https://doi.org/10.1016/j.sna.2014.01.017

16 B. Huang and X. Shu: Opt. Express 26 (2018) 4563. https://doi.org/10.1364/oe.26.004563

17 H. Meng, S. Wei, G. Zhang, X. Wu, W. Wang, C. Tan, and X. Huang: Sens. Actuators, B 160 (2011) 720. https://doi.org/10.1016/j.snb.2011.08.054

18 A. Zhou, Y. Zhang, G. Li, J. Yang, Y. Wang, F. Tian, and L. Yuan: Opt. Lett. 36 (2011) 3221. https://doi. org/10.1364/ol.36.003221

19 Q. Rong, X. Qiao, Y. Du, D. Feng, R. Wang, Y. Ma, H. Sun, M. Hu, and Z. Feng: Appl. Opt. 52 (2013) 1441. https://doi.org/10.1364/AO.52.001441

20 Z. Tian, S. S. Yam, and H. P. Loock: Opt. Lett. 33 (2008) 1105. https://doi.org/10.1364/OL.33.001105

21 T. H. Xia, A. P. Zhang, B. Gu, and J. J. Zhu: Opt. Commun. 283 (2010) 2136. https://doi.org/10.1016/j. optcom.2010.01.031

22 J. Tang, J. Zhou, J. Guan, S. Long, J. Yu, H. Guan, H. Lu, Y. Luo, J. Zhang, and Z. Chen: IEEE J. Sel. Top. Quantum Electron. 23 (2017) 238. https://doi.org/10.1109/JSTQE.2016.2615941

23 H. Wu, L. Yuan, L. Zhao, Z. Cao, and P. Wang: United States: Proc. 2014 SPIE Advanced Fabrication Technologies for Micro/Nano Optics and Photonics VII (SPIE, 2014) 89741D.

24 S. M. A. Musa, N. F. Baharin, A. I. Azmi, R. K. R. Ibrahim, A. S. Abdullah, M. Y. Mohd Noor, and H. Qi: Microw. Opt. Technol. Lett. 60 (2018) 822. https://doi.org/10.1002/mop.31056

25 Y. C. Liang, C. C. Liao, and Y. L. Lo: IEEE Photonics Technol. Lett. 26 (2014) 2330. https://doi.org/10.1109/ LPT.2014.2355262

26 H. Fu, N. Zhao, M. Shao, X. Yan, H. Li, Q. Liu, H. Gao, Y. Liu, and X. Qiao: IEEE Sens. J. 15 (2015) 6869. https://doi.org/10.1109/JSEN.2015.2465165

27 S. Zhang, T. Yuan, and L. Yuan: Korea Proc. 2017 IEEE 25th Int. Conf. Optical Fiber-optic Sensors (IEEE, 2017) 1032377.

28 Y. Zhao, L. Cai, and X. G. Li: IEEE Photonics Technol. Lett. 27 (2015) 1341. https://doi.org/10.1109/ LPT.2015.2421349

29 Q. Wang, L. Kong, Y. Dang, F. Xia, Y. Zhang, Y. Zhao, H. Hu, and J. Li: Sens. Actuators, B 225 (2016) 213. https://doi.org/10.1016/j.snb.2015.11.047

30 Z. Yang, J. Xia, S. Li, R. Qi, G. Zuo, and W. Li: Opt Express 28 (2020) 258. https://doi.org/10.1364/oe.381213

31 L. Xu, L. Jiang, S. Wang, B. Li, and Y. Lu: Appl. Opt. 52 (2013) 2038. https://doi.org/10.1364/AO.52.002038

32 Y. C. Tan, W. B. Ji, V. Mamidala, K. K. Chow, and S. C. Tjin: Sens. Actuators, B 196 (2014) 260. https://doi. org/10.1016/j.snb.2014.01.063

33 B. Xu, Y. Yang, Z. Jia, and D. Wang: Opt. Express 25 (2017) 14483. https://doi.org/10.1364/OE.25.014483

34 Chen. P, X. Shu, and H. Cao: IEEE Photonics J. 9 (2017) 5. https://doi.org/10.1109/JPHOT.2017.2732957

35 Y. Lopez-Dieguez, J. M. Estudillo-Ayala, D. Jauregui-Vazquez, L.A. Herrera-Piad, J. M. Sierra-Hernandez, and J. C. Hernandez-Garcia: IEEE Photonics Technol. Lett. 30 (2018) 15. https://doi.org/10.1109/ LPT.2017.2771409

36 M. Madrigal, D. Barrera, and S. Sales: J. Light Technol. 37 (2019) 18. https://doi.org/10.1109/JLT.2019.2917629 